\title{
Der Brennerverkehrsgipfel-Prozess. Ein Meilenstein der Verkehrspolitik in der Europaregion Tirol - Ein Diskussionsbericht
}

\author{
Stephanie Jicha
}

\section{Einleitung}

Deutschland, Österreich, Italien, Bayern, Nordtirol und Südtirol streben gemeinsame Lösungen für den Verkehr auf der Brennerachse an. Die Regierungsvertreter*innen der Staaten und Regionen entlang der Brennerachse ringen um Lösungen für die Verkehrssituation am Brenner. Ein gemeinsames Ziel ist die Verlagerung des Güterverkehrs von der Straße auf die Schiene.

Elf Millionen Fahrzeuge und über 2,3 Millionen LKW fahren jedes Jahr über den Brenner. Die zunehmende Verkehrsbelastung ist für die Bevölkerung entlang der Transitachse und auch für die Umwelt eine enorme Belastung. Ein großer Lichtblick in der gesamten Transitproblematik ist die Fertigstellung des Brennerbasistunnels (BBT) ${ }^{1}$.

Dass es gemeinsame Lösungen braucht, zeigt der Transitstreit zwischen den Regionen, insbesondere zwischen Bayern und Tirol. Regionale Maßnahmen wirken sich regelmäßig auf die anderen Regionen negativ aus. Die notwendigen LKWBlockabfertigungen in Tirol führen beispielsweise zu Rückstaus im bayrischen Inntal. Tirol spricht von Notwehr, Bayern von Provokation.

Auf zahlreichen Verkehrsgipfeln haben sich die politischen Vertreter*innen der Regionen und Nationalstaaten getroffen und gemeinsam Maßnahmen erarbeitet. Im Fokus dieser Treffen standen kurzfristige Lösungen wie der stetige Ausbau der

${ }^{1}$ Siehe Bergmeister, in diesem Band, Kap. 9.

Article note: Stephanie Jicha ist Vizepräsidentin des Tiroler Landtags. Die Verfasserin bringt in diesem Beitrag allein ihre persönliche Auffassung zum Ausdruck.

\footnotetext{
S. Jicha $(\bowtie)$

Vizepräsidentin des Tiroler Landtages, Innsbruck, Österreich

E-Mail: stephanie.jicha@tirol.gv.at
}

S. Laimer, C. Perathoner (Hrsg.), Mobilitäts- und Transportrecht in Europa,

Bibliothek des Wirtschaftsrechts 2,

https://doi.org/10.1007/978-3-662-63635-0_15 
rollenden Landstraße (RoLa) und eine einheitliche Korridormaut von München bis nach Verona sowie langfristige Maßnahmen wie beispielsweise die Erhöhung des Tempos beim Bau der Zulaufstrecken des BBT.

Anlässlich einer Tagung an der Universität Innsbruck haben Vertreter*innen aus Politik und Wirtschaft über das BBT-Großprojekt diskutiert. ${ }^{2}$ Im Folgenden werden die wesentlichen Aspekte der Debatte zusammengetragen.

\section{Die regionalpolitische Perspektive}

\subsection{Ingrid Felipe}

Landeshauptmann-Stv ${ }^{\text {in }}$ Ingrid Felipe ist seit 2013 in der Tiroler Landesregierung ua ressortzuständig für Verkehr- und Mobilität sowie auch für Umwelt- und Klimaschutz.

In diesen gut sechs Jahren Amtszeit hat sie zahlreiche Wechsel von Kolleg*innen im Bereich der Verkehrspolitik erlebt. Nicht nur in Österreich gab es in dieser Zeit fünf verschiedene Minister, sondern auch in Deutschland und Italien war eine hohe Fluktuation in den Verkehrs- und Infrastrukturressorts zu beobachten. Die Europaregion Tirol, Südtirol, Trentino sowie auch das zuständige Kommissionsmitglied in der Europäischen Kommission waren im Vergleich überdurchschnittlich stabil. Durch die hohe Fluktuation in der Exekutive war die Zusammenarbeit nicht immer ganz leicht.

Die erste Lektion, die Felipe gelernt hat, ist die Notwendigkeit der Nationalstaaten, um die Verkehrsherausforderung über die Brennerachse aber auch in der gesamten EU zu bewältigen. Bei Veranstaltungen war sie oft mit ihrem Kollegen Dr. Mussner aus Südtirol alleine. Weder von den Nationalstaaten noch aus anderen Regionen waren Vertreter*innen anwesend. Es braucht aber dringend die Entscheidungsträger*innen und Exekutivpolitiker*innen an einem Tisch. Das ist mit dem Brennerverkehrsgipfel-Prozess gelungen.

Die zweite Lektion, die Felipe lernen durfte, ist die Notwendigkeit eines Verlagerungsgesetzes. Ein solches fehlt in Österreich und in der EU. Daher müssen alle Maßnahmen auf die Luftreinhaltung und auf die Verkehrssicherheit gestützt werden. Das führt in der Regel zu komplizierten Konstruktionen. Es braucht daher eine Initiative im Gesetzwerdungsprozess, die auf Verlagerung abstellt. Ein hilfreiches Instrument ist die neue Wegekostenrichtlinie. Es können dadurch viele der externen Effekte wie Staukosten, Lärmkosten, Umweltkosten viel besser berücksichtigt werden. Das ist ein wichtiger Schritt in Richtung Kostenwahrheit und fairer Wettbewerb.

Die dritte Lektion ist die Kooperation. Kooperation ist grundlegend, wenn man in der europäischen Verkehrspolitik etwas voranbringen will. Keine Region kann das alleine bewerkstelligen. Die Blockabfertigungen in Tirol haben letztlich dazu

\footnotetext{
${ }^{2}$ Siehe zu der Veranstaltung auch Viertler, Mobilitäts- und Transportrecht in Europa: Bestandsaufnahme und Zukunftsperspektiven - Tagungsbericht, JB1 2019, 432 f.
} 
geführt, dass es zum ersten Brennerverkehrsgipfel in München gekommen ist. Es waren die zuständigen Minister aus Italien und Deutschland da, aber auch der bayrische Verkehrsminister und die Landeshauptleute aus Tirol und Südtirol. Es haben alle eingesehen, dass etwas passieren muss. Der zweite Gipfel in Bozen war dann schon nicht mehr so prominent besetzt und dadurch war auch das Ergebnis nicht mehr zur Freude aller. Da steht der Prozess nun und da muss jetzt weitergearbeitet werden. Der nächste Schritt wäre eine Pilotregion für eine Korridormaut über den Brenner mit den Spielregeln der neuen Wegekostenrichtlinie. Dafür braucht es dringend die Zusammenarbeit in der Europaregion aber natürlich auch mit den Nationalstaaten.

Ein Wendepunkt in der Verkehrspolitik war für Felipe die Akzeptanz des sektoralen Fahrverbotes durch die Kommission und letztlich durch die gesamte EU. Erstmalig wurde mit der Argumentation des Schutzes der Gesundheit der Bevölkerung in der Alpenregion eine Einschränkung des freien Warenverkehrs gestattet. Trotz massiv steigender Verkehrszahlen konnten seit dem Inkrafttreten des sektoralen Fahrverbotes bessere lufthygienische Werte erzielt werden. Es ist bisher aber nicht das optimale Werkzeug, um die Verlagerung voranzutreiben. Deshalb muss da noch nachgeschärft werden. Ein Ziel wäre es den Schutz der Gesundheit der Menschen in den Rechtsrahmen zu implementieren und eine Alpentransitbörse einzurichten.

\subsection{Florian Mussner}

Landesrat a.D. Florian Mussner war von 2001 bis 2018 Mitglied der Südtiroler Landesregierung und von 2013 bis 2018 im Kabinett Kompatscher I ua ressortzuständig für Verkehr und Mobilität.

Wenn es darum geht die Mobilität in Zukunft zu gestalten, dann liegt es für Mussner daran, die Weichen heute richtig zu stellen. Es muss sich jemand an die Arbeit machen, dass die Mobilität von morgen genau so ist wie wir sie haben wollen. Mobilität ist ein Grundbedürfnis unserer Gesellschaft, unserer Wirtschaft und unseres Sozialbereiches und zugleich eine der größten Herausforderungen unserer Zeit. Mobilität bewegt nicht nur Menschen, sondern auch die Gemüter. Mobilität ist komplex und steht wie kaum ein anderes Thema im Fadenkreuz vieler Interessen, vieler Ansprüche und Erwartungen und die können zum Teil auch völlig konträr sein. Einerseits gilt es flächendeckende Mobilität für alle interessierten Gruppen zu ermöglichen, andererseits bringt das mittlerweile ausufernde Verkehrsaufkommen große Belastungen mit sich. Mobilität muss im Einklang stehen mit dem Recht auf Lebensqualität und mit dem Ziel, dass Mensch und Umwelt, Straßen und Ortschaften möglichst wenig belastet werden.

Es muss vermieden, verlagert, verbessert und vernetzt werden. Das ist die Aufgabe der Verantwortlichen für die Mobilitätspolitik im Alpenraum und auch in Europa. Der Alpenraum ist ein besonders sensibles Gebiet. Die Bevölkerung, die den Alpenraum bewohnt, weiß um die Probleme und muss diese deswegen angehen. Zwischen den Landeshauptleuten und den zuständigen Regierungsmitgliedern gab 
es in der Europaregion immer eine gute Kooperation. Die Europaregion hat vorgezeigt, dass eine Öffnung notwendig ist. Im nächsten Schritt müssen Tarife, Mauten, Umweltvorschriften und Datensysteme angeglichen und von allen mitgetragen werden.

Es ist nicht viele Jahre her, dass am BBT gezweifelt wurde bzw ist das jetzt von Rom wieder so zum Ausdruck gekommen. Für Mussner, der 17 Jahre Mitglied der Südtiroler Landesregierung war, ist es unverständlich, dass so etwas in Frage gestellt werden kann. Es fehlen nur mehr 8 Jahre bis das Projekt verwirklicht ist. Bis dahin müssen aber alle vorbereitet sein. Bayern muss aktiver werden so wie es alle anderen gewesen sind und es vorgemacht haben. Wichtig ist, dass mit den Menschen geredet wird, Partizipation stattfindet und alle verantwortlich gemacht werden mitzuarbeiten und mitzureden sodass eine Lösung gefunden werden kann. Das braucht es immer mehr und mehr in Europa, auch in Bayern.

\section{Moderne Perspektiven der Verkehrswirtschaft}

\subsection{Christoph Walser}

Christoph Walser ist seit 2018 Präsident der Wirtschaftskammer Tirol, seit 2016 Bürgermeister von Thaur und leitet gemeinsam mit seiner Frau das Unternehmen CWA Transporte.

Mit fast 30-jähriger Transport- und Speditionserfahrung hat Walser einen sehr guten Einblick in die Realität. Für ihn ist die Transportwirtschaft der Seismograf der Wirtschaft. Innerhalb von wenigen Tagen ist zu bemerken wo die Entwicklung in der Wirtschaft hingeht. In Zeiten in denen die Wirtschaft nicht so gut läuft, ist weniger Verkehr. In Zeiten, wie in den letzten zwei Jahren, in denen die Wirtschaft auf höchstem Niveau läuft, ist mehr Verkehr auf der Straße. Einerseits wird Wirtschaftswachstum gefordert und andererseits gibt es das Problem, dass mehr Fahrzeuge auf der Straße sind. Es ist gemeinsame Aufgabe von Wirtschaftskammer und Land Tirol, dass die Belastung in einem erträglichen Ausmaß stattfindet aber auf der anderen Seite auch der Tiroler Wirtschaft nicht geschadet wird. Es ist mittlerweile jedem verantwortungsvollen Menschen und Politiker auch wichtig, dass bei allen Entscheidungen die Umwelt nicht außer Acht gelassen wird.

Es gibt vieles, das gerade am Laufen ist. Ein positiver Ausfluss der schwarzgrünen Landesregierung ist, dass das Thema der Nachhaltigkeit intensiver und auch offener diskutiert wird. Statt ein Verbot nach dem anderen zu erlassen wird in Gesprächen versucht Lösungen für die Zukunft zu finden. Im Bereich des Nahverkehrs wird man den LKW nie ganz wegbringen. Daher muss intensiv daran gearbeitet werden, dass es schlussendlich zum Einsatz von Elektroantrieb oder Wasserstoffantrieb kommt. Tirol hat große Chancen und könnte, was die Umrüstung auf alternative Antriebsarten betrifft, eine Vorreiterrolle einnehmen. Die Forschung und Wissenschaft könnte sich mit der Wirtschaft verbinden und gute Akzente setzen. Es 
wäre ein interessantes Projekt in Innsbruck zu versuchen den Verteilerverkehr komplett auf Elektroantrieb umzustellen. Ideen gibt es viele. Die Wirtschaftskammer ist jederzeit gesprächsbereit.

Im Bereich der Verlagerung wurden in den letzten Jahren ganz viele Akzente gesetzt. Ein Beispiel ist die rollende Landstraße. Auch der BBT ist sicher ein zentraler Punkt in der Verlagerung von der Straße auf die Schiene betreffend den internationalen Transitverkehr. Tirol und Südtirol machen ihre Hausaufgaben. Nördlich wird es schwieriger wenn die Zulauf- und Ablaufstrecken nicht geregelt sind. Betreffend den BBT gibt sich Walser gerne bewusst kritisch. Er fragt sich wohin sich die Mobilität in nächster Zeit entwickelt. Wenn auf Elektromobilität und Wasserstoff umgerüstet wird, stellt sich die Frage ob der BBT in der Form die richtige Entscheidung war, erst recht wenn der Nordzulauf nicht realisiert wird. Walser fordert intensive Bemühungen dahingehend, dass die Güter dann wirklich mit der Bahn transportiert werden können. Eine weitere Forderung der Tiroler Wirtschaftskammer ist die Investition in Infrastruktur. Die Tiroler Wirtschaft hilft dann sehr gerne mit bei der Verlagerung. Die ÖBB hat in den letzten Jahren sehr viel in den Personenverkehr investiert. Der Güterverkehr wurde aber vernachlässigt. Die Terminals sind veraltet, es gibt zu wenige Lokführer, zu wenige Wagons.

Neben der Verlagerung ist es auch wichtig, dass in die Umrüstung auf alternative Antriebsarten investiert wird. Tirol war da Vorreiter. Es gab Förderungen für die Tiroler Wirtschaft, wenn LKW der Euroklasse 4 und älter gegen neuere LKW ausgetauscht wurden. Betreffend das Nachtfahrverbot, das sektorale Fahrverbot und die Euroklassen-Fahrverbote hält Walser fest, dass es wirtschaftlich notwendig ist Übergangsfristen in der Länge von zumindest der Zeit der Abschreibdauer eines LKW vorzusehen. Gleichzeitig muss in die Entwicklung neuer Fahrzeuge und neuer Modelle investiert werden sowie auch der Ausbau der Bahn vorangetrieben werden.

Die Gesellschaft hat sich gewandelt und die Transportwirtschaft hat sich angepasst. Jeder will um 16:00 Uhr eine Lieferung bestellen und am nächsten Tag um 08:00 Uhr zugestellt bekommen. Diese Lieferung muss von jemandem gemacht werden. Es darf dabei nicht immer eine Seite verdammt werden und die andere Seite, in dessen Auftrag die Transportwirtschaft tätig wird, nicht.

\section{Die Brenner-Verkehrsachse: eine Transitroute als Lebensraum}

\subsection{Renate Gebhard}

Renate Gebhard ist Rechtsanwältin und seit 2013 Abgeordnete zum Römischen Parlament. Sie setzt sich dort insbesondere für die Südtiroler*innen ein.

Als Eisacktalerin ist Gebhard tagtäglich mit der Brennerverkehrsachse konfrontiert. Der BBT ist für sie eine absolute Notwendigkeit. Verkehr ist Fluch und Segen 
zugleich. Alle fahren gerne mal mit dem Auto, um bequem ihr Ziel zu erreichen oder genießen Obst aus anderen Ländern. Der Verkehr hat Wirtschaftswachstum und Wohlstand gebracht. Aber auch Abgase, Lärm und Staus.

Der Brennerpass ist verkehrstechnisch gut gelegen und der niedrigste Übergang über den Alpenhauptkamm. Daher hat er schon früh Reisende und Händler angezogen. Städte und Dörfer entlang der Brennerstrecke florierten. In den 50ern erlebte das Automobil einen Boom und der Verkehr ist angestiegen. Waren es 1950 noch 65.000 Fahrzeuge, die pro Jahr über den Brenner gefahren sind, so waren es 1958 bereits 2 Mio. Die Landstraße war überlastet und die Menschen entlang der Strecke haben bereits damals an den Folgen gelitten. Mit dem Bau einer Gebirgsautobahn auf der Trasse Brenner-Sterzing-Brixen-Bozen konnte die Landstraße entlastet werden. Die Freude an der Brennerautobahn war nur kurz, denn der Verkehr stieg weiter an. Die Belastungsgrenze ist einmal mehr erreicht. Vor 10 Jahren gab es noch ca. fünf sogenannte schwarze Tage an denen es auf der A 22 kein Weiterkommen gab. Heute gibt es von Ostern bis Weihnachten kein staufreies Wochenende mehr. Nicht nur die Autobahn ist blockiert, sondern auch die Landstraße. Auch gibt es keine Zeitfenster mehr für notwendige Instandhaltungsarbeiten. Jede Baustelle führt zu Reklamationen oder einem Verkehrskollaps.

Was früher Wohlstand und Reichtum gebracht hat, schränkt mittlerweile die Lebensqualität stark ein. Die Menschen machen sich Sorgen. Die konstante Lärmbelästigung und die Abgase schädigen die Gesundheit. Politiker*innen sind gefordert Lösungen zur Verbesserung zu suchen. Der Verkehr auf der Autobahn zwischen Verona und Kufstein muss besser koordiniert werden. Bei Feiertagsfahrverboten, Blockabfertigungen, Verkehrsinformationen und Bemautungen muss besser kooperiert werden. Es kann nicht sein, dass wenn in Tirol Blockabfertigungen gemacht werden, sich im Süden die LKW vom Brenner bis nach Trient stauen. Wenn dann noch eine Baustelle dazukommt geht in Südtirol gar nichts mehr.

Betreffend die Mautpolitik fordert Gebhard mehr Spielraum bei der Mautgestaltung. Es kann nicht sein, dass viele LKW nur deswegen über den Brenner fahren, weil der Sprit in Österreich so billig ist und die Maut in Italien nichts kostet. Das produziert unnötigen Umwegverkehr. Wenn die A 22 als Inhouse-Gesellschaft betrieben werden könnte, dann wäre Südtirol unabhängiger vom italienischen Ministerium und es könnten auch Umweltmaßnahmen getroffen werden.

Die Autobahn ist nicht die Lösung. Es braucht auch die Verlagerung des Verkehrs auf die Schiene. Deswegen ist der Bau des BBT unumgänglich sowie auch die Einhaltung der Zeitpläne. Daneben braucht es auch entsprechende verkehrspolitische Maßnahmen wie beispielsweise einen Kostenvorteil für die Schiene. Darüber hinaus ist ein effizientes Verkehrs-, Umwelt- und Gesundheitsmanagement mit einer geeigneten Strategie, geeigneten rechtlichen Rahmenbedingungen sowie Kooperation notwendig. Damit können schrittweise Lösungen für die verkehrsgeplagten Menschen erarbeitet werden, um sowohl den wirtschaftlichen als auch den gesellschaftlichen Interessen gerecht zu werden. 


\section{Mobilität in Tirol: Zukünftige Herausforderungen}

\subsection{Hermann Gahr}

Hermann Gahr ist seit 1999 Abgeordneter zum Nationalrat. Er ist Südtirolsprecher und war jahrelang im Verkehrsausschuss.

Gahr ist es wichtig, dass der Nationalrat sowohl wirtschaftlich als auch verkehrstechnisch seine Hausaufgaben macht. Er hat 20 Jahre lang das Thema Verkehr und Mobilität miterlebt. Für Tirol ist dieses Thema eine Zukunftsfrage. In Österreich gab es immer und gibt es ein klares Bekenntnis zum Ausbau der Bahn und zum BBT. Die Unterinntaltrasse wird bereits genutzt und zeigt, dass das die richtige Entscheidung war. Eine Alternative ist unvorstellbar. Auch damals als die Unterinntaltrasse 97/98 errichtet wurde gab es Verunsicherung in der Bevölkerung. Mit den Menschen muss geredet werden. Diese Überzeugungsarbeit braucht es auch in Bayern. Dort muss etwas passieren, es kann nicht mehr länger gewartet werden. Es braucht keinen Planungsstopp sondern es braucht Tempo was die Trassenplanung betrifft.

Die besten Köpfe werden benötigt, es braucht mutige Konzepte, Innovation und einen gewissen Weitblick. Die Herausforderungen sind ganz klar. Tirol hat sich als Land positiv entwickelt und trotzdem sorgt man sich um die Lebensqualität und um den von Tourismus und wirtschaftlicher Entwicklung geprägten Standort. In der Verkehrsfrage braucht es regionale, nationale und internationale Maßnahmen. Ein Zusammenspiel zwischen Gemeinden, Land, Bund und EU. Es braucht eine Autobahn, die wieder funktioniert. Es muss zwar auch der freie Warenverkehr gewährleistet sein, aber es kann nicht sein, dass der Weg durch Tirol der Billigste ist. Darüber hinaus muss der eigene Wirtschaftsverkehr bewältigt werden und die eigene Mobilität sichergestellt sein.

Auch die Bahn muss attraktiver werden. In den letzten Jahren wurde zwar viel investiert, Bahnhöfe wurden modernisiert aber was die Bahnlogistik und den Gütertransport betrifft, so wurden einige Dinge verabsäumt. Es gibt zu viele Logistiksysteme, die nicht miteinander harmonieren. Die Verkehrsverlagerung gelingt nicht. In Tirol werden ca. 20-30 \% der Güter mit der Bahn transportiert. In der Schweiz bereits ca. $70 \%$. Das könnte für Tirol ein Ziel sein. Und dafür braucht es die EU. Im öffentlichen Personennahverkehr steht Tirol gut da. In den letzten Jahren wurde dieser ernsthaft ausgebaut. Diese positiven Entwicklungen sind der derzeitigen Regierung zu verdanken.

Vergleicht man die Regierungsprogramme auf Bundes- und Landesebene so ist der Bereich Verkehr und Mobilität auf Bundesebene nicht ganz zufriedenstellend. Da sind sehr wenige konkrete Forderungen aufgelistet. Der BBT war einmal das Infrastrukturprojekt Nr. 1. In Wien ist da eine gewisse Kraft verloren gegangen. Das muss noch einmal, insbesondere von Seiten des Landes, verstärkt eingefordert werden. Der Bund bekommt aus dem Land Tirol sehr viele Mauteinnahmen, das Land bekommt aber nicht dieselben Mittel für Verkehrsinfrastruktur zurück. Diese Mittel müssen konsequent eingefordert werden. 
Es gibt einen klaren politischen Auftrag, der nur gemeinsam bewältigt werden kann. Die Bürger*innen wünschen sich Mobilität aber auch Lebensqualität. Es gilt sowohl im Wettbewerb als auch in der Lebensqualität zu bestehen.

\section{Zusammenfassung}

Ingrid Felipe, Florian Mussner, Christoph Walser, Renate Gebhard und Hermann Gahr teilen ihre Gedanken zur europäischen Verkehrspolitik und zum BBT. Eine Forderung, die sich durch alle Beiträge zieht, ist die Notwendigkeit der Kooperation und Zusammenarbeit zwischen den Ländern, den Nationalstaaten und der EU. Auch wollen alle die Verlagerung auf die Schiene forcieren und fordern einen Ausbau der Bahnlogistik. Darüber hinaus besteht ein Bekenntnis zum BBT und es wird von allen Druck auf Bayern ausgeübt, dass alsbald die Zulaufstrecken fertiggestellt werden. Als große Herausforderung sieht ein Großteil der Politiker*innen das Spannungsverhältnis zwischen dem Wirtschaftswachstum und dem Wohlstand auf der einen Seite und der Lebensqualität und der Gesundheit der Menschen auf der anderen Seite.

Open Access Dieses Kapitel wird unter der Creative Commons Namensnennung 4.0 International Lizenz (http://creativecommons.org/licenses/by/4.0/deed.de) veröffentlicht, welche die Nutzung, Vervielfältigung, Bearbeitung, Verbreitung und Wiedergabe in jeglichem Medium und Format erlaubt, sofern Sie den/die ursprünglichen Autor(en) und die Quelle ordnungsgemäß nennen, einen Link zur Creative Commons Lizenz beifügen und angeben, ob Änderungen vorgenommen wurden.

Die in diesem Kapitel enthaltenen Bilder und sonstiges Drittmaterial unterliegen ebenfalls der genannten Creative Commons Lizenz, sofern sich aus der Abbildungslegende nichts anderes ergibt. Sofern das betreffende Material nicht unter der genannten Creative Commons Lizenz steht und die betreffende Handlung nicht nach gesetzlichen Vorschriften erlaubt ist, ist für die oben aufgeführten Weiterverwendungen des Materials die Einwilligung des jeweiligen Rechteinhabers einzuholen.

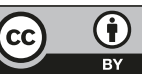

